\title{
Carfilzomib/pomalidomide single-agent or in combination with other agents for the management of relapsed/refractory multiple myeloma: a meta-analysis of 37 trials
}

\author{
Yandun Zou ${ }^{1}$, Xiaoyan Ma ${ }^{2}$, Haiying $\mathrm{Yu}^{3}$, Chunling Hu${ }^{1}$, Limei Fan ${ }^{1}$ and Xuehong \\ $\operatorname{Ran}^{4}$ \\ ${ }^{1}$ Internal Medicine, Guang Dong Women and Children Hospital, Guang Zhou, China \\ 2 Department of I.C.U, Weifang People's Hospital, Weifang, China \\ ${ }^{3}$ Department of Pediatrics, Weifang People's Hospital, Weifang, China \\ ${ }^{4}$ Department of Hematology, Weifang People's Hospital, Weifang, China \\ Correspondence to: Xuehong Ran, email: shengzhixin5569@126.com
}

Keywords: pomalidomide, carfilzomib, lenalidomide, bortezomib, multiple myeloma

Received: April 25, $2016 \quad$ Accepted: July 10, $2016 \quad$ Published: July 21, 2016

Copyright: Zou et al. This is an open-access article distributed under the terms of the Creative Commons Attribution License (CC-BY), which permits unrestricted use, distribution, and reproduction in any medium, provided the original author and source are credited.

\section{ABSTRACT}

Purpose: The use of carfilzomib/pomalidomide single-agent or in combination with other agents in patients with refractory/relapsed multiple myeloma (RRMM) was not clearly clarified in clinical practice. We sought to compile the available clinical reports to better understand the efficacy and safety of carfilzomib (CFZ) and pomalidomide (POM).

Results: Based on our research criteria, we identified 37 prospective studies that evaluated 1160 patients. Analysis of subgroup differences between carfilzomib single-agent and CFZ/DEX dual combination showed significantly $\left(P<0.001, \mathrm{I}^{2}=\right.$ $96.3 \%$ ), suggesting the overall response rate (ORR) of $66 \%$ attained from CFZ/DEX dual combination seemed to be higher than that of $\mathbf{2 8 \%}$ from carfilzomib single-agent. And, the same trend favoring CFZ/DEX dual combination was found in $\geq$ VGPR and CBR analysis. The ORR of $31 \%$ attained from POM/DEX dual combination was superior to that of $19 \%$ from pomalidomide single-agent $\left(P<0.001, \mathrm{I}^{2}=94.4 \%\right)$. And, the same trend favoring POM/DEX dual combination was found in $\geq$ VGPR and CBR analysis. However, the ORR of $83 \%$ attained from POM/BOR/DEX triplet combination was superior to that of $31 \%$ from POM/DEX dual combination $\left(P<0.001, \mathrm{I}^{2}=99.1 \%\right)$. And, the same trend favoring POM/BOR/DEX triplet combination was found in $\geq V G P R$ analysis.

Methods: We searched published reports including carfilzomib and (or) pomalidomide therapy for RRMM who had received bortezomib and (or) lenalidomide.

Conclusion: Pomalidomide/Carfilzomib plus dexamethasone seemed to attain a superior response rate compared with pomalidomide/carfilzomib single-agent. Furthermore, the combination of pomalidomide, bortezomib and dexamethasone resulted in a much higher response rate compared with pomalidomide plus dexamethasone regimen. These results needed more validation in future trials.

\section{INTRODUCTION}

In the past decades, the administration of novel agents (thalidomide lenalidomide and bortezomib) had produced a pronounced shift in the treatment framework for myeloma patients. And, treatment options and corresponding patient outcomes had greatly improved because of them. However, myeloma still remained incurable, and most patients would ultimately relapse and resist these agents [1]. Relapsed disease was characterized by increasingly lower remission rate even following salvage therapy. And, survival among those in whom 
lenalidomide, bortezomib, and thalidomide have failed was especially poor [2]. So, there was still an urgent need for new treatments to improve outcomes for these patients with RRMM.

Pomalidomide was a potent immunomodulatory drug that was FDA-approved for treatment of patients with RRMM. Preclinical studies had shown pomalidomide had robust antiangiogenic, antiapoptotic, and tumor necrosis factor-a inhibitory activity, stimulating antibodydependent cytotoxic T-cell activity. Pomalidomide single-agent had been found to be active in relapsed/ refractory patients $[3,4]$. Preclinical studies had shown pomalidomide plus dexamethasone (DEX) had synergistic antiproliferative effects in LEN-resistant myeloma cells [5]. This pomalidomide dual regimen (POM/DEX) had been found to be active in several clinical trials with participants with RRMM [4, 6-13]. Meanwhile, several trials had shown pomalidomide triplet combinations (CFZ-POM-DEX, BOR-POM-DEX, CYC-POM-DEX et al.) also were effective for patients with RRMM [13-23].

Meanwhile, several trials had demonstrated the activity of carfilzomib single, dual and triplet combination regimens [17, 18, 24-41]. However, which strategy would be the optimal therapy for patients with RRMM still remains undefined. Furthermore, these published reports consisted of the clinical trials with small sample sizes, and these small trials were not enough power to determine the efficacy and safety of pomalidomide and carfilzomib. The present pooled analysis highlighted the two most recently approved anti-MM agents, carfilzomib and pomalidomide, and looked ahead toward optimal regimens in patients with RRMM.

\section{RESULTS}

\section{Characteristics of the published reports of carfilzomib and pomalidomide}

Based on our research criteria, we identified 37 prospective studies of carfilzomib and pomalidomide enrolling a total of 3432 patients with RRMM [13-21]. Of them, nine evaluated outcomes from carfilzomib single-agent [24-32]; eleven evaluated outcomes from carfilzomib combination regimens $[17,18,31,33-41]$; two evaluated outcomes from pomalidomide single-agent $[3,4]$; sixteen evaluated outcomes from pomalidomide combination regimens [4, 6-23].The characteristics of these trials were shown in Table 1.

\section{Response rate to carfilzomib single-agent and carfilzomib combination regimens}

Finally, nine trials enrolling a total of 957 patients evaluated the treatment effects on overall response of carfilzomib single-agent for the management of patients with RRMM. As shown in Figure 1, pooled analysis showed ORR was $28 \%$ of carfilzomib singleagent. Eleven trials enrolling a total of 1169 patients evaluated the treatment effects on overall response of carfilzomib combination regimens in patients with RRMM. Carfilzomib combination regimens resulted into an impressive ORR of $61 \%$ (Figure 1), which was higher than that of $28 \%$ from carfilzomib single-agent $(P<0.001$, $I^{2}=97.1 \%$ ) (Table 2).

In order to strengthen the reliability of this pooled analysis and decrease the heterogeneity, we undertook subgoup analysis based on carfilzomib regimens (singleagent, CFZ/DEX dual combination, CFZ/LEN/DEX triplet combination), as shown in Figure 2 and Table 2. Analysis of subgroup differences between carfilzomib single-agent and CFZ/DEX dual combination showed significantly $(P$ $<0.001, \mathrm{I}^{2}=96.3 \%$ ), suggesting the overall response rate (ORR) of $66 \%$ attained from CFZ/DEX dual combination seems to be higher than that of $28 \%$ from carfilzomib single-agent. And, the same trend favoring CFZ/DEX dual combination in $\geq \mathrm{VGPR}$ and $\mathrm{CBR}$ analysis. CFZ/LEN/ DEX triplet combination resulted into a similar response outcome to that from CFZ/DEX dual combination therapy in ORR, $\geq$ VGPR, CBR and SDR analysis(Table 2). And, $\mathrm{CFZ/POM/DEX} \mathrm{triplet} \mathrm{combination} \mathrm{had} \mathrm{a} \mathrm{similar} \mathrm{response}$ outcome to that from CFZ/DEX dual combination therapy in ORR, $\geq$ VGPR, and CBR analysis(Table 2).

\section{Response rate to pomalidomide single-agent and pomalidomide combination regimens}

Finally, two trials enrolling a total of 146 patients evaluated the treatment effects on overall response of pomalidomide single-agent for the management of patients with RRMM. As shown in Figure 1, pooled analysis showed ORR was $19 \%$ of pomalidomide singleagent. Sixteen trials enrolling a total of 1160 patients evaluated the treatment effects on overall response of pomalidomide combination regimens in patients with RRMM. Pomalidomide combination regimens resulted into an impressive ORR of 45\%(Figure 1), which was higher than that of $19 \%$ from pomalidomide single-agent $\left(P<0.001, I^{2}=97.9 \%\right)$ (Table 2$)$.

We undertook subgoup analysis based on pomalidomide regimens (single-agent, POM/DEX dual combination, POM/BOR/DEX triplet combination), as shown in Figure 2 and Table 2. Analysis of subgroup differences between pomalidomide single-agent and POM/ DEX dual combination showed significantly $(P<0.001$, $\left.\mathrm{I}^{2}=94.4 \%\right)$, suggesting the overall response rate (ORR) of $31 \%$ attained from POM/DEX dual combination seems to be higher than that of $19 \%$ from carfilzomib singleagent. And, the same trend favoring POM/DEX dual combination in $\geq V G P R$ and $\mathrm{SDR}$ analysis. $\mathrm{POM} / \mathrm{BOR} /$ 
Rate Ratio

Rate Ratio

\begin{tabular}{|c|c|c|c|c|c|c|}
\hline \multirow{2}{*}{\multicolumn{4}{|c|}{$\begin{array}{l}\text { Study or Subgroup log[Rate Ratio] } \\
\text { 1.1.1 ORR from Carfilzomib Single-agent }\end{array}$}} & \multicolumn{3}{|c|}{ IV, Random, 95\% Cl } \\
\hline & & & & & & \\
\hline Badros 2013 & -1.36649173 & 0.06357689 & $0.25[0.23,0.29]$ & $=$ & & \\
\hline Hajek R 2015 & -1.65548185 & 0.03137192 & $0.19[0.18,0.20]$ & - & & \\
\hline Jagannath 2012 & -1.78976147 & 0.05755143 & $0.17[0.15,0.19]$ & - & & \\
\hline Jakubowiak 2013 & -1.39070407 & 0.02857219 & $0.25[0.24,0.26]$ & - & & \\
\hline Lendvai 2014 & -0.48955349 & 0.08748338 & $0.61[0.52,0.73]$ & $\rightarrow$ & & \\
\hline Papadopoulos 2015 & -0.72360639 & 0.08699965 & $0.49[0.41,0.58]$ & $=$ & & \\
\hline Siegel 2012 & -1.43969514 & 0.02652589 & $0.24[0.22,0.25]$ & - & & \\
\hline Vij 2012 -1 & -1.76609172 & 0.06364163 & $0.17[0.15,0.19]$ & $=$ & & \\
\hline Vij $2012-2$ & -0.74233742 & 0.0444922 & $0.48[0.44,0.52]$ & - & & \\
\hline Subtotal $(95 \% \mathrm{Cl})$ & & & $0.28[0.22,0.36]$ & & & \\
\hline \multirow{2}{*}{\multicolumn{7}{|c|}{$\begin{array}{l}\text { Heterogeneity: } \operatorname{Tau}^{2}=0.12 ; \mathrm{Chi}^{2}=534.89, \mathrm{df}=8(\mathrm{P}<0.00001) ; \mathrm{I}^{2}=99 \% \\
\text { Test for overall effect: } Z=10.67(\mathrm{P}<0.00001)\end{array}$}} \\
\hline & & & & & & \\
\hline \multicolumn{7}{|c|}{ 1.1.2 ORR from Carfilzomib Combinations } \\
\hline Berdeja 2015 & -0.40496523 & 0.07272109 & $0.67[0.58,0.77]$ & $=$ & & \\
\hline Berenson $2014-2$ & -0.40496523 & 0.12168566 & $0.67[0.53,0.85]$ & $=$ & & \\
\hline Berenson 2014-1 & -0.83932969 & 0.08143577 & $0.43[0.37,0.51]$ & - & & \\
\hline Dimopoulos 2015 & -0.26266431 & 0.01956637 & $0.77[0.74,0.80]$ & - & - & \\
\hline Kaufman 2014 & -0.69314718 & 0.1118034 & $0.50[0.40,0.62]$ & $\rightarrow$ & & \\
\hline Niesvizky 2013 & -0.47000363 & 0.07654655 & $0.63[0.54,0.73]$ & - & & \\
\hline Papadopoulos 2015 & -0.60696948 & 0.10616775 & $0.55[0.44,0.67]$ & $\rightarrow$ & & \\
\hline Shah 2015 & -0.69314718 & 0.08838835 & $0.50[0.42,0.59]$ & $\rightarrow$ & & \\
\hline Stewart 2015 & -0.1381133 & 0.01684443 & $0.87[0.84,0.90]$ & & - & \\
\hline Vesole 2015 & -0.63676685 & 0.12106367 & $0.53[0.42,0.67]$ & $\rightarrow$ & & \\
\hline Wang 2013 & -0.37106368 & 0.05046215 & $0.69[0.63,0.76]$ & $=$ & & \\
\hline Subtotal $(95 \% \mathrm{Cl})$ & & & $0.61[0.55,0.69]$ & 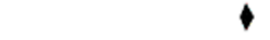 & & \\
\hline \multicolumn{7}{|c|}{ Heterogeneity: $\operatorname{Tau}^{2}=0.03 ; \mathrm{Chi}^{2}=179.45, \mathrm{df}=10(\mathrm{P}<0.00001) ; I^{2}=94 \%$} \\
\hline \multicolumn{7}{|c|}{ Test for overall effect: $Z=7.89(P<0.00001)$} \\
\hline \multicolumn{7}{|c|}{ 1.1.3 ORR from Pomalidomide Single agent } \\
\hline Richardson 2013-1 & -1.56064775 & 0.06607412 & $0.21[0.18,0.24]$ & 口 & & \\
\hline Richardson 2014 & -1.71479843 & 0.03696846 & $0.18[0.17,0.19]$ & $\mathbf{\square}$ & & \\
\hline Subtotal $(95 \% \mathrm{Cl})$ & & & $0.19[0.17,0.22]$ & $\bullet$ & & \\
\hline \multicolumn{7}{|c|}{ Heterogeneity: $\mathrm{Tau}^{2}=0.01 ; \mathrm{Chi}^{2}=4.15, \mathrm{df}=1(\mathrm{P}=0.04) ; \mathrm{I}^{2}=76 \%$} \\
\hline \multicolumn{7}{|c|}{ Test for overall effect: $Z=21.55(P<0.00001)$} \\
\hline \multicolumn{7}{|c|}{ 1.1.4 ORR from Pomalidomide Combinations } \\
\hline Baz 2016-A & -0.69314718 & 0.15811388 & $0.50[0.37,0.68]$ & $\rightarrow$ & & \\
\hline Baz 2016-B & -0.94160854 & 0.08129166 & $0.39[0.33,0.46]$ & - & & \\
\hline Baz 2016-C & -0.43078292 & 0.08179961 & $0.65[0.55,0.76]$ & $\rightarrow$ & & \\
\hline Lacy 2009 & -0.46203546 & 0.06232977 & $0.63[0.56,0.71]$ & - & & \\
\hline Lacy 2010 & -1.12701176 & 0.08026134 & $0.32[0.28,0.38]$ & $\rightarrow$ & & \\
\hline Lacy $2011-2 \mathrm{mg}$ & -1.35812348 & 0.07387642 & $0.26[0.22,0.30]$ & - & & \\
\hline Lacy $2011-4 \mathrm{mg}$ & -1.25276297 & 0.07636036 & $0.29[0.25,0.33]$ & - & & \\
\hline Lacy 2014 & -0.16251893 & 0.05208422 & $0.85[0.77,0.94]$ & & + & \\
\hline Larocca 2013 & -0.67334455 & 0.0674065 & $0.51[0.45,0.58]$ & - & & \\
\hline Leleu 2013 & -1.06421086 & 0.05186693 & $0.34[0.31,0.38]$ & + & & \\
\hline Leleu 2015 & -1.51412773 & 0.05858327 & $0.22[0.20,0.25]$ & & & \\
\hline Mark 2013 & -0.48776035 & 0.04559586 & $0.61[0.56,0.67]$ & - & & \\
\hline Matsue 2015 & -1.38629436 & 0.125 & $0.25[0.20,0.32]$ & $\rightarrow$ & & \\
\hline Miguel 2013 & -1.15518264 & 0.02672988 & $0.32[0.30,0.33]$ & - & & \\
\hline Mikhael 2013 & -0.18632958 & 0.10039209 & $0.83[0.68,1.01]$ & $\rightarrow$ & $\rightarrow$ & \\
\hline Richardson 2013-2 & -0.28768207 & 0.09682458 & $0.75[0.62,0.91]$ & $\rightarrow$ & - & \\
\hline Richardson 2014 & -1.11779511 & 0.04413084 & $0.33[0.30,0.36]$ & - & & \\
\hline Shah 2013 & -0.4462871 & 0.05864133 & $0.64[0.57,0.72]$ & - & & \\
\hline Shan $20152 / 7$ & -0.53062825 & 0.11936462 & $0.59[0.47,0.74]$ & $\rightarrow$ & & \\
\hline Shan $20155 / 14$ & -0.69314718 & 0.25 & $0.50[0.31,0.82]$ & & & \\
\hline Subtotal $(95 \% \mathrm{Cl})$ & & & $0.45[0.37,0.54]$ & $\boldsymbol{\nabla}$ & & \\
\hline \multirow{2}{*}{\multicolumn{7}{|c|}{$\begin{array}{l}\text { Heterogeneity: } \text { Tau }^{2}=0.17 ; \mathrm{Chi}^{2}=855.53, \mathrm{df}=19(\mathrm{P}<0.00001) ; \mathrm{I}^{2}=98 \% \\
\text { Test for overall effect: } Z=8.38(\mathrm{P}<0.00001)\end{array}$}} \\
\hline & & & & & & \\
\hline & & & & 0.1 & 10 & 100 \\
\hline
\end{tabular}

Figure 1: Meta-analysis of the overall response rate (ORR) of carfilzomib/pomalidomide single agent and combination regimens in patients with relapsed and refractory multiple myeloma. $n$, number of the enrolled patients. CI, $95 \%$ confidence interval. Random, random-effects model. 
Table 1: Characteristics of included studies

\begin{tabular}{|c|c|c|c|c|c|c|c|c|c|c|c|c|c|}
\hline \multirow{2}{*}{\multicolumn{2}{|c|}{$\begin{array}{l}\text { Author, year } \\
\text { Strategy }\end{array}$}} & \multirow{2}{*}{$\begin{array}{l}\text { Age } \\
\text { (M) }\end{array}$} & \multirow{2}{*}{$\begin{array}{l}\mathbf{F} / \mathbf{M} \\
(\mathbf{n} / \mathbf{N})\end{array}$} & \multirow{2}{*}{$\begin{array}{l}\text { TFD } \\
\text { (Y) } \\
\text { (M) }\end{array}$} & \multirow{2}{*}{$\begin{array}{l}\text { Cytogenetic } \\
\text { F/U/M }\end{array}$} & \multirow{2}{*}{ Pom/CFZ schedule } & \multirow{2}{*}{$\begin{array}{l}\text { Prior } \\
\text { therapy } \\
\text { (M) }\end{array}$} & \multicolumn{2}{|c|}{ Prior therapy } & \multirow{2}{*}{ Regimen } & \multirow{2}{*}{ ORR } & \multirow{2}{*}{$\begin{array}{l}\text { PFS } \\
(\mathrm{m})\end{array}$} & \multirow{2}{*}{$\begin{array}{l}\text { OS } \\
(\mathrm{m})\end{array}$} \\
\hline & & & & & & & & Bort & Lena & & & & \\
\hline \multicolumn{14}{|c|}{ Pomalidomide Trials } \\
\hline \multicolumn{2}{|c|}{ Richardson 2014 [4] } & 61 & $55 / 53$ & & $43 / 30 / 35$ & $21 / 28(4 \mathrm{mg})$ & $5(1-12)$ & 76 & 86 & Poma alone & $21 \%$ & 9.5 & 30 \\
\hline \multicolumn{2}{|c|}{ Richardson 2013-1 [3] } & 66 & $20 / 18$ & 5.5 & - & $21 / 28(4 \mathrm{mg})$ & $6(2-17)$ & 28 & 31 & Poma alone & $18 \%$ & 4.6 & 18.3 \\
\hline \multicolumn{2}{|c|}{ Miguel 2013 [6] } & 64 & $121 / 181$ & 5.3 & -- & $21 / 28(4 \mathrm{mg})$ & $5(2-14)$ & 302 & 302 & Pom+LoDex & $32 \%$ & 4.0 & 12.7 \\
\hline \multirow{2}{*}{\multicolumn{2}{|c|}{ Lacy 2011 [7] }} & 62 & $8 / 27$ & 5.2 & $15 /-/-$ & $28 / 28(2 \mathrm{mg})$ & - & 35 & 35 & Pom+LoDex & $26 \%$ & 6.5 & $78 \%(6 \mathrm{~m})$ \\
\hline & & 61 & $14 / 21$ & 6.0 & $21 /-/-$ & $28 / 28(4 \mathrm{mg})$ & - & 35 & 35 & Pom+LoDex & $29 \%$ & 3.2 & $67 \%(6 \mathrm{~m})$ \\
\hline Lacy & $10[8]$ & 61.5 & $11 / 23$ & 5.2 & $14 / 20 /-$ & $28 / 28(2 \mathrm{mg})$ & - & 20 & 34 & Pom+LoDex & $32 \%$ & 4.8 & 13.9 \\
\hline Richa & son 2014 [4] & 64 & $58 / 55$ & - & $31 / 56 / 26$ & $21 / 28(4 \mathrm{mg}$ & $5(1-13)$ & 113 & 113 & Pom+LoDex & $33 \%$ & 4.2 & 16.5 \\
\hline I & 012 Г0] & 60 & -- & 5.1 & $8 / 15 / 10$ & $21 / 28(4 \mathrm{mg})$ & $5(1-13)$ & 34 & 36 & Pom+LoDex & $34 \%$ & 5.4 & 14.9 \\
\hline Lereu & $013[9]$ & 60 & -- & 6.5 & $13 / 19 / 9$ & $28 / 28(4 \mathrm{mg})$ & $5(2-10)$ & 34 & 39 & Pom+LoDex & & 3.7 & 14.8 \\
\hline Lacy & 09 [10] & 65.5 & $24 / 36$ & 3.6 & $19 / 38 / 2$ & $28 / 28(2 \mathrm{mg})$ & - & 20 & 21 & Pom+LoDex & $63 \%$ & 11.6 & $76 \%(2 y)$ \\
\hline Leleu & 015 [11] & 63 & $20 / 30$ & 3 & $60 / 0 / 0$ & $21 / 28(4 \mathrm{mg})$ & $3(1-10)$ & 48 & 50 & Pom+LoDex & $22 \%$ & 2.8 & 12 \\
\hline Matsu & $2015[12]$ & 68 & $5 / 7$ & 5.15 & - & $21 / 28(2 \mathrm{mg}, 4 \mathrm{mg})$ & $6.0(4-10)$ & 12 & 12 & Pom+LoDex & $25 \%$ & 5.5 & \\
\hline Baz 2 & 6-B [13] & 64 & $13 / 23$ & - & - & $21 / 28(4 \mathrm{mg})$ & $4(2-12)$ & 28 & - & Pom+LoDex & $39 \%$ & 4.4 & 16.8 \\
\hline Baz 2 & $6-\mathrm{A}[13]$ & 69 & $3 / 7$ & - & - & $21 / 28(4 \mathrm{mg})$ & $5(4-12)$ & 10 & - & PCD & $50 \%$ & - & - \\
\hline Baz 2 & 6-C [13] & 65 & $16 / 18$ & - & - & $21 / 28(4 \mathrm{mg})$ & $4(2-9)$ & 24 & - & PCD & $65 \%$ & 9.5 & NR \\
\hline Shah & 15 5/14 [14] & 57 & - & - & - & $21 / 28(4 \mathrm{mg})$ & $8(2-22)$ & - & - & OPD & $50 \%$ & - & - \\
\hline Shah & $152 / 7$ [14] & 66 & - & - & - & - & $4.5(2-11)$ & - & - & OPD & $59 \%$ & - & - \\
\hline Mark & 2012 [15] & 65 & $25 / 21$ & - & $20 / 24$ & $28 / 28(4 \mathrm{mg})$ & $5(3-15)$ & 42 & 46 & ClaPD & $61 \%$ & 8.13 & $85 \%(9.4 \mathrm{~m})$ \\
\hline Mark & 2013 [16] & - & - & - & - & $28 / 28(4 \mathrm{mg})$ & $5(3-15)$ & 90 & 97 & ClaPD & & 8.1 & - \\
\hline Shah & \begin{tabular}{|l}
$2012 / 2015$ \\
{$[17,18]$}
\end{tabular} & 63.5 & $12 / 20$ & 5.0 & $18 / 6 / 5 / 1$ & $21 / 28(4 \mathrm{mg})$ & $6(1-15)$ & 31 & 32 & CPD & $64 \%$ & 7.4 & - \\
\hline Shah & 2013 [19] & 64 & $27 / 45$ & 5.1 & & $21 / 28(4 \mathrm{mg})$ & $6(2-15)$ & 62 & 67 & CPD & & 12 & 16.3 \\
\hline Laroc & 2013 [20] & 69 & $27 / 28$ & 53 & $31 / 13 / 11$ & $21 / 28(2.5 \mathrm{mg})$ & $3(1-3)$ & 46 & 55 & PCP & $51 \%$ & 10.4 & $73 \%(14.8 \mathrm{~m})$ \\
\hline Lacy & $14[21]$ & 66 & $24 / 23$ & 49 & $4 / 38 / 5$ & $21 / 28(4 \mathrm{mg})$ & $2(1-5)$ & 27 & 47 & PVD & $85 \%$ & 10.7 & - \\
\hline Mikhe & 12013 [22] & 66 & $8 / 8$ & 45 & - & $21 / 28(4 \mathrm{mg})$ & $3(1-6)$ & 8 & 16 & PVD & $83 \%$ & - & - \\
\hline Richa & son 2013-2 [23] & 57 & - & - & - & $14 / 21(4 \mathrm{mg})$ & $2(1-4)$ & 21 & 21 & PVD & $75 \%$ & - & - \\
\hline Carfil & mib Trials & & & & & & & & & & & & \\
\hline Vij 20 & $2-1[24]$ & 63 & $17 / 18$ & 3.6 & $25 / 9 / 1$ & $20 \mathrm{mg}$ & $3.0(1-13)$ & 35 & 13 & CFZ alone & $17 \%$ & $4.6 \mathrm{~m}$ & $29.9 \mathrm{~m}$ \\
\hline $\mathrm{Vij} 20$ & $2-2[25]$ & 65 & $53 / 76$ & 3.6 & $103 / 19 / 7$ & $20 / 27 \mathrm{mg}$ & $2(1-4)$ & 3 & 76 & CFZ alone & $48 \%$ & $54.3 \%(9 \mathrm{~m})$ & - \\
\hline Siegel & 012 [26] & 63 & $111 / 155$ & 5.4 & $159 / 75 / 32$ & $20 / 27 \mathrm{mg}$ & $5(1-20)$ & 265 & 249 & CFZ alone & $24 \%$ & 3.7 & 15.6 \\
\hline Jagan & th $2012[27]$ & 63.5 & $21 / 25$ & 5.5 & $33 / 7 / 5$ & $20 \mathrm{mg}$ & $5(2-16)$ & 46 & 42 & CFZ alone & $17 \%$ & $3.5 \mathrm{~m}$ & - \\
\hline Jakub & viak 2013 [28 ] & 65 & 76/91 & 5.6 & - & $20 / 27 \mathrm{mg}$ & $5(1-20)$ & 166 & - & CFZ alone & $25 \%$ & 4.6 & 19 \\
\hline & & 63 & $21 / 41$ & 5.3 & - & $20 / 27 \mathrm{mg}$ & $5(2-12)$ & 62 & - & CFZ alone & & 3.5 & 9.3 \\
\hline Badro & 2013 [29] & 64 & $22 / 28$ & 6.3 & $32 / 13 / 5$ & $15 / 20 / 27 \mathrm{mg}$ & $5(1-15)$ & 48 & 44 & CFZ alone & $25 \%$ & - & - \\
\hline Hajek & 2015 [30 ] & 65 & $11 / 22$ & 4.7 & $22 / 7 / 4$ & $20 / 36 / 45 / 56 / 70 \mathrm{mg}$ & $5(1-9)$ & 30 & - & CFZ alone & $19 \%$ & 3.7 & 10.2 \\
\hline $\begin{array}{l}\text { Papad } \\
{[31]}\end{array}$ & oulos 2015 & 63.3 & $75 / 82$ & - & - & $20 / 27 \mathrm{mg}$ & - & - & - & CFZ alone & $49 \%$ & 7.0 & - \\
\hline Lendv & $2014[32]$ & 63 & $25 / 19$ & - & $23 / 20 / 1$ & $20 / 56 \mathrm{mg}$ & $5(1-11)$ & 44 & - & CFZ alone & $61 \%$ & 4.1 & 20.3 \\
\hline Kaufn & n 2014[40] & 64.5 & - & - & - & $20 / 36 / 45 \mathrm{mg}$ & - & - & - & $\mathrm{CP}$ & $50 \%$ & 14.3 & - \\
\hline Berde & 2015 [33] & 66 & $27 / 17$ & - & - & $20 / 27 / 36 / 45 \mathrm{mg}$ & $5(1-10)$ & - & - & $\mathrm{CP}$ & $67 \%$ & 7.7 & $67 \%(24 m)$ \\
\hline Papad & poulos 2015[31] & 59.5 & $5 / 17$ & 3.6 & $14 / 7 / 1$ & $20 / 36 / 45 / 56 / 70 \mathrm{mg}$ & $4(2-9)$ & 21 & - & $\mathrm{Cd}$ & $55 \%$ & 6.2 & - \\
\hline Beren & n 2014-2[38] & 63 & - & - & - & $20 / 45 / 56 / 70 / 88 \mathrm{mg}$ & $1(1-2)$ & - & - & $\mathrm{Cd}$ & $67 \%$ & - & - \\
\hline Dimo & ulos 2015[39] & - & - & - & - & $20 / 56 \mathrm{mg}$ & - & - & - & $\mathrm{Cd}$ & $77 \%$ & 18.7 & - \\
\hline Niesv & ky 2013[35] & 61.5 & $18 / 22$ & 3.3 & $25 / 11 / 4$ & $15 / 20 / 27 \mathrm{mg}$ & $2(1-3)$ & 30 & 28 & CRd & $63 \%$ & 10.2 & - \\
\hline Stewa & $2015[36]$ & 64 & $181 / 215$ & 3.0 & $48 / 147 / 201$ & $20 / 27 \mathrm{mg}$ & $2(1-3)$ & 261 & 79 & CRd & $87 \%$ & 26.3 & $73.3 \%(2 y)$ \\
\hline Wang & $013[37]$ & 61.5 & $36 / 48$ & 3.1 & $57 / 22 / 5$ & $20 / 27 \mathrm{mg}$ & $2(1-5)$ & 65 & 59 & CRd & $69 \%$ & 15.4 & - \\
\hline Shan2 & $12 / 2015[17,18]$ & 64 & $12 / 20$ & 5.9 & $10 /-/-$ & $20 / 27 / 36 / 45 / 56 \mathrm{mg}$ & $6(2-12)$ & 31 & 32 & CPD & $50 \%$ & 7.2 & 20.6 \\
\hline Vesol & 2015 [41] & 61 & $7 / 10$ & 4 & $3 / 12 / 2$ & $15 / 20 / 27 \mathrm{mg}$ & $4(1-9)$ & 17 & 16 & QUAD & $53 \%$ & 12 & NR \\
\hline Beren & n 2014-1 [34] & 67 & $13 / 25$ & 4.2 & - & $20 / 27 / 36 / 45 \mathrm{mg}$ & - & - & - & & $43 \%$ & 8.3 & 15.8 \\
\hline
\end{tabular}

Abbreviations: F female; M male; (M) median; TFD time from diagnosis; F/U/M Favor/Unfavor/miss; Bor bortezomib; Lena lenalidomide; Pom pomalidomide;Dex Low dose dexamethasone; PCD pomalidomide cyclophosphamide and dexamethasone;OPD Oprozomib, Pomalidomide, and Dexamethasone; ClaPD Clarithromycin pomalidomide, and dexamethasone, CPD Carfilzomib, pomalidomide, and dexamethasone; PCP pomalidomide cyclophosphamide and prednisone; PVD pomalidomide bortezomib and dexamethasone; CP Carfilzomib, panobinostat; Cd Carfilzomib, dexamethasone; CRd Carfilzomib, lenalidomide , and dexamethasone; CPD Carfilzomib, pomalidomide, and dexamethasone; QUAD carfilzomib, lenalidomide, vorinostat and dexamethasone; Replacement of bortezomib with carfilzomib from bortezomib combination therapy; ORR overall response rate; NR: Not reach; $54.3 \%(9 \mathrm{~m}) 54.3 \%$ progression free at 9 months; $78 \%(6 \mathrm{~m}) 78 \%$ alive at 6 months; $67 \%(6 \mathrm{~m}) 67 \%$ alive at $6 \mathrm{months} ; 76 \%(2 \mathrm{y}) 67 \%$ alive at 2 years; $85 \%(9.4 \mathrm{~m}) 85 \%$ alive at 9.4 months; $73 \%(14.8 \mathrm{~m}) 73 \%$ alive at 14.8 months; $67 \%(24 \mathrm{~m}) 67 \%$ alive at $24 \mathrm{months} ; 73.3 \%(2 \mathrm{y}) 73.3 \%$ alive at 2 years. 
Table 2: Summary of Response Outcomes from Carfilzomib and Pomalidomide

\begin{tabular}{|c|c|c|c|c|c|c|c|c|c|}
\hline Outcomes & CFZ strategy & $\begin{array}{l}\text { Num of } \\
\text { Studies }\end{array}$ & RR $(95 \%$ CI) & $\begin{array}{l}\text { Test for subgroup } \\
\text { heterogeneity }\end{array}$ & Outcomes & POM strategy & $\begin{array}{l}\text { Num of } \\
\text { Studies }\end{array}$ & RR $(95 \%$ CI $)$ & $\begin{array}{l}\text { Test for subgroup } \\
\text { heterogeneity }\end{array}$ \\
\hline \multicolumn{5}{|c|}{ CFZ sing-agent versus CFZilzomib combination regimens } & \multicolumn{5}{|c|}{ POM sing-agent versus POM combination regimens } \\
\hline \multirow{2}{*}{ ORR } & A:CFZ single-agent & 9 & $0.28[0.22,0.36]$ & $\begin{array}{l}\mathrm{A} \\
\mathrm{BI}^{2}=97.1 \%, \mathrm{P}<0.001\end{array}$ & \multirow{2}{*}{ ORR } & A:POM single-agent & 2 & $0.19[0.17,0.22]$ & $\begin{array}{ll}\mathrm{A} & \mathrm{V} \\
\mathrm{BI}^{2}=97.9 \%, \mathrm{P}<0.001\end{array}$ \\
\hline & B:CFZ combinations & 11 & $0.61[0.55,0.69]$ & & & $\begin{array}{l}\text { B:POM } \\
\text { combinations }\end{array}$ & 16 & $\begin{array}{l}0.45 \quad[0.37, \\
0.54] \quad \text { } \\
\end{array}$ & \\
\hline \multirow{2}{*}{$\geq$ VGPR } & A:CFZ single-agent & 4 & $0.10[0.06,0.15]$ & $\begin{array}{l}\mathrm{A} \\
\mathrm{BI}^{2}=95.6 \%, \mathrm{P}<0.001\end{array}$ & \multirow{2}{*}{$\geq \mathrm{VGPR}$} & A:POM single-agent & 2 & $0.02[0.02,0.04]$ & $\begin{array}{ll}\mathrm{A} & \mathrm{V} \\
\mathrm{BI}^{2}=97.2 \%, \mathrm{P}<0.001\end{array}$ \\
\hline & B:CFZ combinations & 9 & $0.34[0.25,0.46]$ & & & $\begin{array}{l}\text { B:POM } \\
\text { combinations }\end{array}$ & 15 & $0.15[0.10,0.24]$ & \\
\hline \multirow{2}{*}{ CBR } & A:CFZ single-agent & 8 & $0.37[0.31,0.44]$ & $\begin{array}{l}\mathrm{A} \\
\mathrm{BI}^{2}=97.8 \%, \mathrm{P}<0.001\end{array}$ & \multirow{2}{*}{ CBR } & A:POM single-agent & 2 & $\begin{array}{ll}0.36 & \quad[0.27, \\
0.48] & \\
\end{array}$ & $\begin{array}{l}\mathrm{A} \\
\mathrm{BI}^{2}=82.1 \%, \mathrm{P}=0.02\end{array}$ \\
\hline & B:CFZ combinations & 9 & $0.76[0.69,0.84]$ & & & $\begin{array}{l}\text { B:POM } \\
\text { combinations }\end{array}$ & 12 & $\begin{array}{l}0.54 \quad[0.46, \\
0.64] \quad\end{array}$ & \\
\hline \multirow{2}{*}{ SD } & A:CFZ single-agent & 7 & $0.31[0.25,0.39]$ & $\begin{array}{l}\mathrm{A} \\
\mathrm{BI}^{2}=90.5 \%, \mathrm{P}=0.001\end{array}$ & \multirow{2}{*}{$\mathrm{SD}$} & A:POM single-agent & 1 & \begin{tabular}{|l|l|}
0.48 & $\quad[0.44$, \\
$0.53]$ & \\
\end{tabular} & $\begin{array}{l}\mathrm{A} \\
\mathrm{BI}^{2}=76.1 \%, \mathrm{P}=0.04\end{array}$ \\
\hline & B:CFZ combinations & 8 & $0.15[0.11,0.22]$ & & & $\begin{array}{l}\text { B:POM } \\
\text { combinations }\end{array}$ & 13 & $\begin{array}{ll}0.28 & {[0.17,} \\
0.47] & \\
\end{array}$ & \\
\hline \multicolumn{5}{|c|}{ CFZ sing-agent versus dual combinations versus triplet combinations } & \multicolumn{5}{|c|}{ POM sing-agent versus dual combinations versus triplet combinations } \\
\hline \multirow{4}{*}{ ORR } & A:CFZ single-agent & 9 & $0.28[0.22,0.36]$ & $\begin{array}{l}\mathrm{A} \\
\mathrm{I}^{2}=96.3 \%, \mathrm{P}<0.001\end{array}$ & \multirow{3}{*}{ ORR } & A:POM single-agent & 2 & $0.19[0.17,0.22]$ & $\begin{array}{lr}\mathrm{A} & \mathrm{V} \\
\mathrm{B}: \mathrm{I}^{2}=95.1 \%, \mathrm{P}<0.001\end{array}$ \\
\hline & $\mathrm{B}: \mathrm{CFZ}+\mathrm{DEX}$ & 3 & $0.66[0.53,0.83]$ & B V C: $I^{2}=0 \%, P=0.57$ & & $\mathrm{~B}: \mathrm{POM}+\mathrm{DEX}$ & 9 & $\begin{array}{l}0.31 \quad[0.26, \\
0.36]\end{array}$ & $\begin{array}{l}\mathrm{B} \\
\mathrm{I}^{2}=99.1 \%, \mathrm{P}<0.001\end{array}$ \\
\hline & $\mathrm{C}: \mathrm{CFZ}+\mathrm{LEN}+\mathrm{DEX}$ & 3 & $0.73[0.59,0.90]$ & B V D: $\mathrm{I}^{2}=0 \%, \mathrm{P}=0.77$ & & $\mathrm{C}: \mathrm{POM}+\mathrm{BOR}+\mathrm{DEX}$ & 3 & $\begin{array}{ll}0.83 & \quad[0.76, \\
0.90] & \\
\end{array}$ & \begin{tabular}{|l}
$\mathrm{B}$ \\
$\mathrm{I}^{2}=98.1 \%, \mathrm{P}<0.001$
\end{tabular} \\
\hline & $\mathrm{D}: \mathrm{CFZ}+\mathrm{POM}+\mathrm{DEX}$ & 1 & $0.64[0.57,0.72]$ & & & $\mathrm{D}: \mathrm{POM}+\mathrm{CFZ}+\mathrm{DEX}$ & 1 & $\begin{array}{l}0.64 \quad[0.57, \\
0.72] \quad \quad \\
\end{array}$ & \\
\hline \multirow{4}{*}{$\geq$ VGPR } & A:CFZ single-agent & 4 & $0.10[0.06,0.15]$ & $\begin{array}{lr}\text { A } & \mathrm{V} \\
\mathrm{B}: \mathrm{I}^{2}=96.3 \%, \mathrm{P}<0.001\end{array}$ & \multirow{3}{*}{$\geq$ VGPR } & A:POM single-agent & 2 & $0.02[0.02,0.04]$ & $\begin{array}{lr}\mathrm{A} & \mathrm{V} \\
\mathrm{B}: \mathrm{I}^{2}=92.7 \%, \mathrm{P}<0.001\end{array}$ \\
\hline & $\mathrm{B}: \mathrm{CFZ}+\mathrm{DEX}$ & 3 & $0.37[0.23,0.61]$ & B V C: $\mathrm{I}^{2}=0 \%, \mathrm{P}=0.51$ & & $\mathrm{~B}: \mathrm{POM}+\mathrm{DEX}$ & 7 & $\begin{array}{l}0.09 \quad[0.05, \\
0.16] \quad\end{array}$ & $\begin{array}{l}\mathrm{B} \\
\mathrm{C}: \mathrm{I}^{2}=95.3 \%, \mathrm{P}<0.001\end{array}$ \\
\hline & $\mathrm{C}: \mathrm{CFZ}+\mathrm{LEN}+\mathrm{DEX}$ & 3 & $0.46[0.29,0.74]$ & $\begin{array}{lcc}\mathrm{B} & \mathrm{V} & \mathrm{D}: \\
\mathrm{I}^{2}=3.8 \%, \mathrm{P}=0.31 & \end{array}$ & & $\begin{array}{l}\text { C:POM+BOR } \\
+ \text { DEX }\end{array}$ & 3 & \begin{tabular}{|l|}
$0.43 \quad[0.30$, \\
$0.60]$
\end{tabular} & $\begin{array}{l}\mathrm{B} \\
\mathrm{I}^{2}=90.5 \%, \mathrm{P}=0.001\end{array}$ \\
\hline & $\mathrm{D}: \mathrm{CFZ}+\mathrm{POM}+\mathrm{DEX}$ & 1 & $0.28[0.25,0.32]$ & & & $\mathrm{D}: \mathrm{POM}+\mathrm{CFZ}+\mathrm{DEX}$ & 1 & $\begin{array}{ll}0.28 & {[0.25,} \\
0.32] & \end{array}$ & \\
\hline \multirow{4}{*}{ CBR } & A:CFZ single-agent & 8 & $0.37[0.31,0.44]$ & \begin{tabular}{|lr}
$\mathrm{A}$ & $\mathrm{V}$ \\
$\mathrm{B}: \mathrm{I}^{2}=93.4 \%, \mathrm{P}<0.001$ \\
\end{tabular} & \multirow{3}{*}{$\mathrm{CBR}$} & A:POM single-agent & 2 & $\begin{array}{ll}0.36 \\
0.48] \quad[0.27, \\
\end{array}$ & $\begin{array}{l}\mathrm{A} \\
\mathrm{B}: \mathrm{I}^{2}=41.1 \%, \mathrm{P}=0.19\end{array}$ \\
\hline & $\mathrm{B}: \mathrm{CFZ}+\mathrm{DEX}$ & 2 & $0.75[0.55,1.01]$ & B V C: $I^{2}=0 \%, P=0.63$ & & $\mathrm{~B}: \mathrm{POM}+\mathrm{DEX}$ & 6 & $\begin{array}{ll}0.44 \quad[0.39, \\
0.50] & \\
\end{array}$ & $\begin{array}{l}\mathrm{B} \\
\mathrm{I}^{2}=98.6 \%, \mathrm{~V}<0.001\end{array}$ \\
\hline & $\mathrm{C}: \mathrm{CFZ}+\mathrm{LEN}+\mathrm{DEX}$ & 3 & $0.81[0.70,0.94]$ & B V D: $\mathrm{I}^{2}=0 \%, \mathrm{P}=0.61$ & & $\begin{array}{l}\text { C:POM+BOR } \\
+ \text { DEX }\end{array}$ & - & & \\
\hline & $\mathrm{D}: \mathrm{CFZ}+\mathrm{POM}+\mathrm{DEX}$ & 1 & $0.81[0.74,0.89]$ & & & $\mathrm{D}: \mathrm{POM}+\mathrm{CFZ}+\mathrm{DEX}$ & 1 & $\begin{array}{l}0.81 \quad[0.74, \\
0.89] \quad\end{array}$ & \\
\hline \multirow{4}{*}{ SDR } & A:CFZ single-agent & 7 & $0.31[0.25,0.39]$ & A V B : $\mathrm{I}^{2}=0 \%, \mathrm{P}=0.34$ & SDR & A:POM single-agent & 1 & \begin{tabular}{|l|l|}
0.48 & {$[0.44$,} \\
$0.53]$ &
\end{tabular} & $\begin{array}{l}\mathrm{A} \\
\mathrm{B}: \mathrm{I}^{2}=82.7 \%, \mathrm{P}=0.02\end{array}$ \\
\hline & $\mathrm{B}: \mathrm{CFZ}+\mathrm{DEX}$ & 2 & $0.15[0.03,0.68]$ & B V C: $\mathrm{I}^{2}=0 \%, \mathrm{P}=0.86$ & & $\mathrm{~B}: \mathrm{POM}+\mathrm{DEX}$ & 9 & $\begin{array}{ll}0.37 & {[0.30,} \\
0.45] & \\
\end{array}$ & \\
\hline & $\mathrm{C}: \mathrm{CFZ}+\mathrm{LEN}+\mathrm{DEX}$ & 3 & $0.13[0.08,0.21]$ & & & $\begin{array}{l}\text { C:POM+BOR } \\
+ \text { +DEX }\end{array}$ & - & & \\
\hline & D:CFZ+POM+DEX & - & & & & D:POM+CFZ+DEX & - & & \\
\hline
\end{tabular}

Abbreviations: CFZ carfilzomib; RR response rate; POM pomalidomide; ORR overall response rate ( $\geq \mathrm{PR})$; $\geq$ VGPR at least very good partial response; CBR clinical benefit rate; SDR stable disease rate; DEX dexamethasone; LEN lenalidomide; BOR bortezomib; A V B Test for subgroup heterogeneity between A and B; B V C Test for subgroup heterogeneity

DEX triplet combination resulted into a superior ORR of $83 \%$ to that of $31 \%$ from POM/DEX dual combination $(P$ $<0.001, I^{2}=99.1 \%$ ) (Table 2). And, a similar trend favoring POM/BOR/DEX triplet combination in $\geq V G P R$ and SDR analysis.

\section{Adverse events}

AEs were outlined in Figure 3-4. Treatment was well tolerated. In the pooled analysis, The most common AEs from carfilzomib single-agent consisted primarily of anemia ( $21 \%$ grade $3 / 4,44 \%$ all grades), thrombocytopenia ( $21 \%$ grade $3 / 4,35 \%$ all grades) and neutropenia ( $8 \%$ grade $3 / 4,15 \%$ all grades), fatigue $(57 \%$ all grades) , nausea (45\% all grades), diarrhea (30\% all grades) , dyspnea (34\% all grades) , pyrexia (33\% all grades), vomitting (28\% all grades) . The most common AEs from carfilzomib combination regimens consisted primarily of anemia (19\% grade $3 / 4,44 \%$ all grades), thrombocytopenia (30\% grade $3 / 4,44 \%$ all grades) and neutropenia (20\% grade $3 / 4,28 \%$ all grades), fatigue (41\% all grades), nausea ( $18 \%$ all grades), diarrhea $(23 \%$ 
all grades) , dyspnea (26\% all grades) , pyrexia (29\% all grades), vomitting ( $6 \%$ all grades) . Notably, there was no significant difference in these AEs analysis between carfilzomib single-agent and carfilzomib combination subgroup, except for neutropenia, nausea, vomiting.

The most common AEs from pomalidomide singleagent consisted primarily of anemia ( $23 \%$ grade $3 / 4,33 \%$ all grades), thrombocytopenia (20\% grade $3 / 4,24 \%$ all grades) and neutropenia (49\% grade 3/4, 54\% all grades), fatigue $(27 \%$ all grades $)$, pneumonia (11\% grade $3 / 4)$, acute renal failure $(6 \%$ grade $3 / 4)$.

The most common AEs from pomalidomide combination regimens consisted primarily of anemia (18\% grade $3 / 4,51 \%$ all grades), thrombocytopenia ( $16 \%$ grade $3 / 4,38 \%$ all grades) and neutropenia (39\% grade $3 / 4,42 \%$ all grades), fatigue ( $43 \%$ all grades) , pneumonia $(9 \%$ grade $3 / 4)$, acute renal failure (5\% grade $3 / 4)$. Notably, there was no significant difference in these AEs analysis between pomalidomide single-agent and pomalidomide combination subgroup, except for anemia, fatigue.

\section{DISCUSSION}

Although the proteasome inhibitor bortezomib was effective to treat myeloma patients, there were still some limits to the use of bortezomib, including occurrence of resistance and neuropathy. So, there was still a need for a second generation of proteasome inhibitors with greater efficacy and less toxicity. Carfilzomib was a potent and highly selective proteasome inhibitor, which seletively and irreversibly inhibits the chymotrypsin-like activity of the $20 \mathrm{~S}$ proteasome. The efficacy and safety of singleagent carfilzomib had been evaluated in a series of phase 2 studies in patients with R/RMM. Because the number
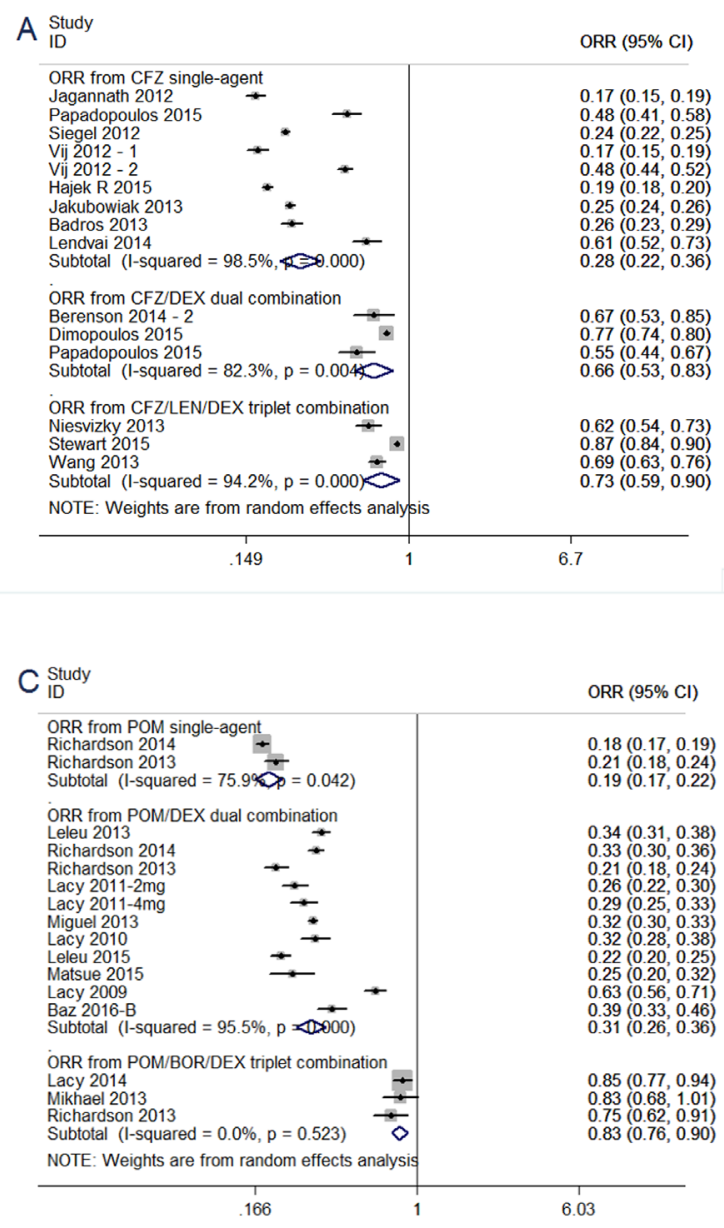
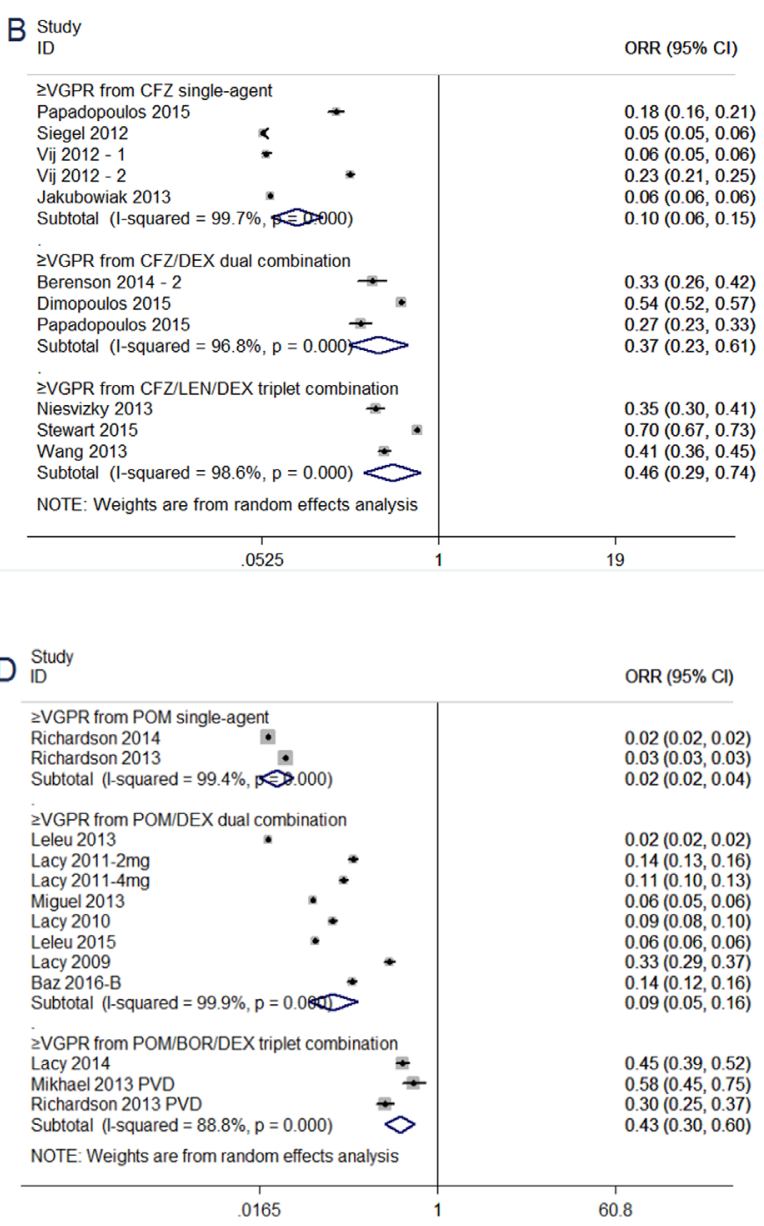

Figure 2: Meta-analysis of the response rate of carfilzomib/pomalidomide single agent, dual and triplet combination regimens in patients with relapsed and refractory multiple myeloma. (A) Overall response rate of carfilzomib single-agent, CFZ/DEX dual combination, CFZ/LEN/DEX triplet combination. (B) At least very good partial response rate of carfilzomib single-agent, CFZ/DEX dual combination, CFZ/LEN/DEX triplet combination. (C) Overall response rate of pomalidomide single-agent, POM/DEX dual combination, POM/BOR/DEX triplet combination. (D) At least very good partial response rate of single-agent, POM/DEX dual combination, POM/BOR/DEX triplet combination. CI, 95\% confidence interval. Random, random-effects model. 


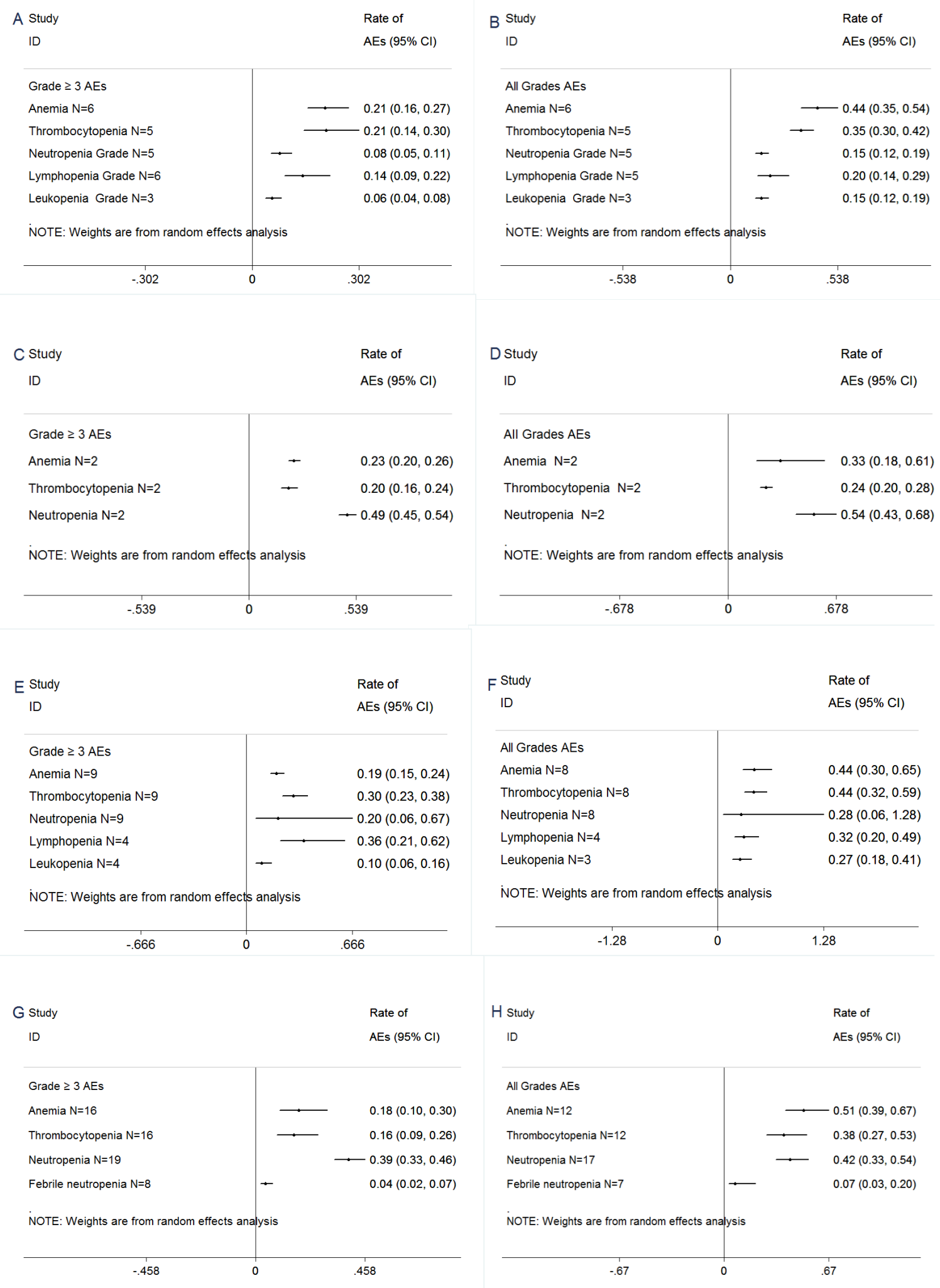

Figure 3: Meta-analysis of hematologic adverse events (AEs) with carfilzomib and pomalidomide for the management of patients with RRMM.. (A) $\geq$ Grade 3 hematologic AEs with carfilzomib single-agent. (B) All grades hematologic AEs with carfilzomib single-agent. (C) $\geq$ Grade 3 hematologic AEs with pomalidomide single-agent.. (D) All grades hematologic AEs with pomalidomide singleagent. (E) $\geq$ Grade 3 hematologic AEs with carfilzomib combinations.(F) All grades hematologic AEs with carfilzomib combinations.(G) $\geq$ Grade 3 hematologic AEs with pomalidomide combinations.(H) All grades hematologic AEs with pomalidomide combinations. 

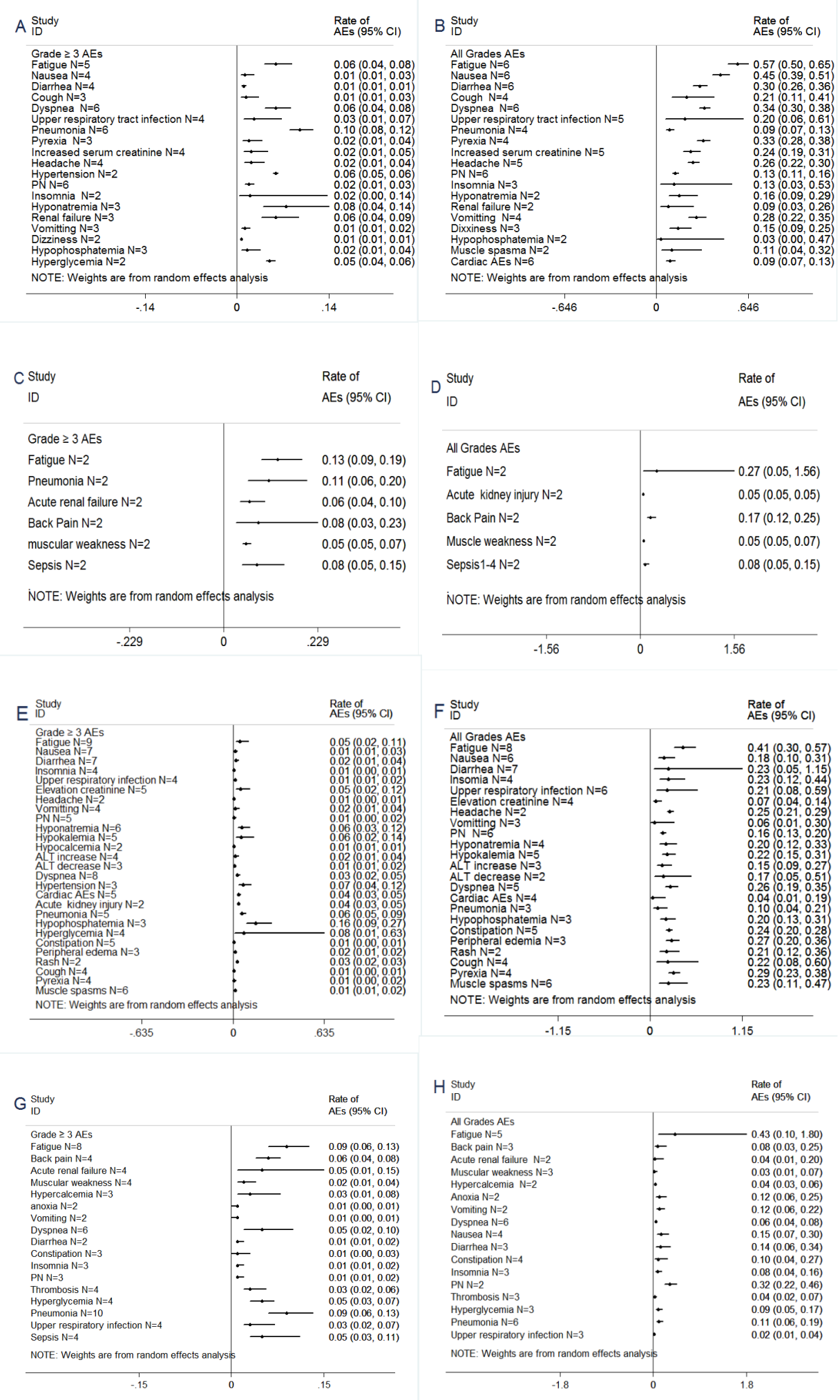

Figure 4: Meta-analysis of non-hematologic adverse events (AEs) with carfilzomib and pomalidomide for the management of patients with RRMM. (A) $\geq$ Grade 3 non-hematologic AEs with carfilzomib single-agent. (B) All grades nonhematologic AEs with carfilzomib single-agent. (C) $\geq$ Grade 3 non-hematologic AEs with pomalidomide single-agent. (D) All grades non-hematologic AEs with pomalidomide single-agent. (E) $\geq$ Grade 3 non-hematologic AEs with carfilzomib combinations.(F) All grades non-hematologic AEs with carfilzomib combinations. $(\mathbf{G}) \geq$ Grade 3 non-hematologic AEs with pomalidomide combinations. (H) All grades non-hematologic AEs with pomalidomide combinations. 
of patients enrolled in these trials was relatively small, we did this pooled analysis. In this aggregated analysis, the best ORR of single-agent carfilzomib for the response evaluable population was $28.0 \%$ and the CBR was $37.0 \%$. These results reinforce the efficacy with carfilzomib monotherapy for a significant number of heavily pretreated patients. The minimal off-target activity characteristic and minimal neurotoxicity of carfilzomib supported it's use in combination with other agent. Pooling three trials of CFZ/DEX dual combination regimen in the 501 relapsed/refractory patients, the $66 \%$ ORR attained with CFZ/DEX dual combination regimen was impressive, particularly when considering the $28 \%$ ORR achieved with single-agent carfilzomib in a similar population with $\mathrm{R} / \mathrm{RMM}$. Furthermore, there was no significant difference between the CFZ/DEX dual and CFZ/LEN/DEX triplet combination in ORR, $\geq$ VGPR,CBR,SDR analysis. So, CFZ/DEX dual regimen still should be good option for patients with RRMM.

Carfilzomib single agent was generally tolerable, with the majority of patients receiving the planned dose. The most common treatment-related AEs were gastrointestinal (nausea, diarrhoea, vomiting, and constipation), fatigue, dyspnea, and myelosuppression (thrombocytopenia, anaemia, and neutropenia). Peripheral neuropathy(PN) was reported infrequently. Comparison of tolerability between carfilzomib and bortezomib containing combination regimens should also be made with caution, but the difference in peripheral neuropathy was notable. Rates of grade $\geq 2 \mathrm{PN}$ were $6.3 \%$ from CFZ/ DEX dual combination $v$ s $32.0 \%$ from POM/DEX dual combination $(P<.0001)$ in ENDEAVOR trial [39]. The result was very encouraging, because PN was the main reason leading to discontinuation of bortezomib.

Preclinical study had shown that the efficacy of pomalidomide might be enhanced by the addition of dexamethasone. This current study pooled from 9 prospective trials of refractory multiple myeloma who received pomalidomide dual combination $(\mathrm{POM}+\mathrm{LoDEX})$ after failure of lenalidomide and bortezomib therapy, and strengthened the individual observations of each of these small prospective studies alone. The ORRs of 31\% with POM+LoDEX was impressive, which compared favorably with $19 \%$ in the POM alone arm (Figure 2, Table 2), and $10 \%$ in the DEX alone [46], in consistent with the synergistic action of POM+LoDEX as observed in previous in vitro studies [47].And, the States Food and Drug Administration already approved pomalidomide in combination with dexamethasone in February2013 for patients with relapsed and refractory multiple myeloma. Recently, there were several trials of pomalidomide triplet combinations. When pooling three trials of $\mathrm{POM} / \mathrm{BOR} /$ DEX triplet combination, an high ORR of $83 \%$ was achieved, which was superior to that of $31 \%$ (Table 2). This finding was impressive, and needed further validation in future trials.
When interpreting our results, there were some caveats that should be considered. The first and major problem was that we used abstracted data, whereas an individual patient data-based meta-analysis might define more clearly treatment efficacy of carfilzomib and pomalidomide. Secondly, as was often the case with metaanalysis, the effect of heterogeneity needed to be taken into account. Although all studies were discussed about the objective response of carfizlomib and pomalidomide after disease progression on lenalidomide and (or) bortezomib, the inclusion criteria were different among individual studies.

Pomalidomide/Carfilzomib plus dexamethasone seemed to attain a superior response rate compared with pomalidomide/carfilzomib single-agent. Furthermore, the combination of pomalidomide, bortezomib and dexamethasone resulted in a much higher response rate compared with pomalidomide plus dexamethasone regimen. These results required validation in future.

\section{MATERIALS AND METHODS}

\section{Literature search strategy}

Medline, Embase, the Cochrane controlled trials register, the Science Citation Index, Conference proceedings from the American Society of Hematology(ASH), the European Hematology association (EHA) and the American Society of Clinical Oncology were searched for trials using the medical subject headings "myeloma", "carfilzomib", "pomalidomide", "bortezomib" and "lenalidomide". Reference lists from studies selected for this review, and from other published systematic reviews and practice guidelines were also handsearched. The study was approved by the institutional review boards of Weifang People's Hospital,in accordance with the Helsinki Declaration.

\section{Selection of studies}

Studies were eligible for inclusion in the metaanalysis if they met all the following criteria: (1) They were published up to February, 2016 and written in English. (2) They dealt only with patients with refractory or relapsed multiple myeloma who had received bortezomib and (or) lenalidomide. (3) Study selection included the setting of these trials: carfilzomib/ pomalidomide single-agent, dual and triplet combination regimens. (4) We included studies that provided sufficient information to allow the calculation of response rate. Multiple reports of a single study were considered as one publication, and only the most recent or complete article was examined. All potentially relevant articles were reviewed by two independent investigators (X.H.R and 
Y.D.Z.).

\section{Outcome measures}

The primary objective of the study was to determine the overall response rate $(\mathrm{ORR}=\geq \mathrm{PR})$, at least very good partial response (VGPR), clinical benefit rate $(\mathrm{CBR}=\geq \mathrm{MR})$, stable disease rate $(\mathrm{SDR})$, progressive disease rate (PDR) of pomalidomide dual and triplet combination regimens, and the secondary objectives were to evaluate the safety of pomalidomide combinations in this population. Responses were investigator assessed based on modified European Group for Bone Marrow Transplantation criteria [42, 43] and International Myeloma Working Group uniform response criteria [44]. National Cancer Institute Common Toxicity Criteria (NCICTC) was used to grade adverse events (AEs).

\section{Statistical analysis}

A random-effects model was used for all the analyses, which incorporates the variability of results among trials and provided a more conservative estimate of an effect size by producing greater confidence intervals (CIs) [45]. We tested for heterogeneity of between-study with the Cochrane $\chi^{2}$ test and quantified its extent with the $I^{2}$ statistic. If significant heterogeneity existed, it would be appropriate to pool the data using random-effects model, but not fixed-effect model. All meta-analyses were completed using Stata ver. 12.0 software (College Station, TX) and Review Manager (version 5.3; Th e Cochrane Collaboration, Oxford, England). Statistical significance was defined as a $\mathrm{P}$ value of less than 0.05 for all tests.

\section{ACKNOWLEDGMENTS}

We are indebted to Yanhua Sun for assistance with data analysis and critiquing the manuscript.

\section{CONFLICTS OF INTEREST}

The authors declare no potential conflicts of interest, and did not receive any financial support.

\section{GRANT SUPPORT}

The authors did not receive any financial support.

\section{REFERENCES}

1. Kumar SK, Lee JH, Lahuerta JJ, Morgan G, Richardson PG, Crowley J, Haessler J, Feather J, Hoering A, Moreau P, LeLeu X, Hulin C, Klein SK, et al. Risk of progression and survival in multiple myeloma relapsing after with IMiDs and bortezomib: A multicenter international myeloma working group study. Leukemia. 2012;26:149-157.

2. Kumar SK, Therneau TM, Gertz MA, Lacy MQ, Dispenzieri A, Rajkumar SV, Fonseca R, Witzig TE, Lust JA, Larson DR, Kyle RA, Greipp PR. Clinical course of patients with relapsed multiple myeloma. Mayo Clin Proc. 2004;79867-874.

3. Richardson PG, Siegel D, Baz R, Kelley SL, Munshi NC, Laubach J, Sullivan D, Alsina M, Schlossman R, Ghobrial IM, Doss D, Loughney N, McBride L, et al. Phase 1 study of pomalidomide MTD, safety, and efficacy in patients with refractory multiple myeloma who have received lenalidomide and bortezomib. Blood. 2013;121:1961-7.

4. Richardson PG, Siegel DS, Vij R, Hofmeister CC, Baz R, Jagannath S, Chen C, Lonial S, Jakubowiak A, Bahlis N, Song K, Belch A, Raje N, et al. Pomalidomide alone or in combination with low-dose dexamethasone in relapsed and refractory multiple myeloma: a randomized phase 2 study. Blood. 2014;123:1826-32.

5. Rychack E, Mendy D, Miller K. Overcoming resistance; the use of pomalidomide (POM) and dexamethasone (DEX) in re-sensitizing lenalidomide (LEN)-resistant multiple myeloma (MM) cells. Haematologica. 2011;96:S126. Abstract P-328.

6. San Miguel J, Weisel K, Moreau P, Lacy M, Song K, Delforge M, Karlin L, Goldschmidt H, Banos A, Oriol A, Alegre A, Chen C, Cavo M, et al. Pomalidomide plus lowdose dexamethasone versus high-dose dexamethasone alone for patients with relapsed and refractory multiple myeloma (MM-003): a randomised, open-label, phase 3 trial. Lancet Oncol. 2013;14:1055-66.

7. Lacy MQ, Allred JB, Gertz MA, Hayman SR, Short KD, Buadi F, Dispenzieri A, Kumar S, Greipp PR, Lust JA, Russell SJ, Dingli D, Zeldenrust S, et al. Pomalidomide plus low-dose dexamethasone in myeloma refractory to both bortezomib and lenalidomide: comparison of 2 dosing strategies in dual-refractory disease. Blood. 2011;118:29705.

8. Lacy MQ, Hayman SR, Gertz MA, Short KD, Dispenzieri A, Kumar S, Greipp PR, Lust JA, Russell SJ, Dingli D, Zeldenrust S, Fonseca R, Bergsagel PL, et al. Pomalidomide (CC4047) plus low dose dexamethasone (Pom/dex) is active and well tolerated in lenalidomide refractory multiple myeloma (MM). Leukemia. 2010;24:1934-9.

9. Leleu X, Attal M, Arnulf B, Moreau P, Traulle C, Marit G, Mathiot C, Petillon MO, Macro M, Roussel M, Pegourie B, Kolb B, Stoppa AM, et al. Pomalidomide plus low-dose dexamethasone is active and well tolerated in bortezomib and lenalidomide-refractory multiple myeloma: Intergroupe Francophone du Myélome 2009-02. Blood. 2013;121:196875.

10. Lacy MQ, Hayman SR, Gertz MA, Dispenzieri A, Buadi F, Kumar S, Greipp PR, Lust JA, Russell SJ, Dingli D, Kyle RA, Fonseca R, Bergsagel PL, et al. Pomalidomide (CC4047) plus low-dose dexamethasone as therapy for 
relapsed multiple myeloma. J Clin Oncol. 2009;27:5008-14.

11. Leleu X, Karlin L, Macro M, Hulin C, Garderet L, Roussel M, Arnulf B, Pegourie B, Kolb B, Stoppa AM, Brechiniac S, Marit G, Thielemans B, et al. Pomalidomide plus lowdose dexamethasone in multiple myeloma with deletion $17 \mathrm{p}$ and/or translocation $(4 ; 14)$ : IFM 2010-02 trial results. Blood. 2015;125:1411-7.

12. Matsue K, Iwasaki H, Chou T, Tobinai K, Sunami K, Ogawa Y, Kurihara M, Midorikawa S, Zaki M, Doerr $\mathrm{T}$, Iida S. Pomalidomide alone or in combination with dexamethasone in Japanese patients with refractory or relapsed and refractory multiple myeloma.Cancer Sci. 2015;106:1561-7.

13. Baz RC, Martin TG, Lin HY, Zhao X, Shain KH, Cho HJ, Wolf JL, Mahindra A, Chari A, Sullivan DM, Nardelli LA, Lau K, Alsina M, et al. Randomized multicenter phase II study of pomalidomide, cyclophosphamide, and dexamethasone in relapsed refractory myeloma. Blood. 2016.

14. Shah J, Niesvizky R, Stadtmauer E, Rifkin RM, Berenson J, Berdeja JG, Sharman JP, Lyons R, Klippel Z, Wong H, Chang YH, Usmani S. Oprozomib, Pomalidomide, and Dexamethasone (OPomd) in Patients (Pts) with Relapsed and/or Refractory Multiple Myeloma (RRMM): Initial Results of a Phase 1b Study (NCT01999335). Blood. 2015;126.

15. Tomer M, Melissa R, Manan S, Ryann Q, Jessica C, Ramsey A. ClaPD (Clarithromycin/[Biaxin $\left.{ }^{\circledR}\right]$, Pomalidomide, Dexamethasone) Therapy in Relapsed or Refractory Multiple Myeloma. Blood. 2012.

16. Tomer M Mark, Angelique Boyer, Adriana C Rossi, Dennis Kwon, Roger N Pearse, Faiza Zafar, Karen Pekle, Linda Tegnestam, David Jayabalan, Scott A Ely, Morton Coleman,Selina Chen-Kiang, Ruben Niesvizky. ClaPD (clarithromycin, pomalidomide, dexamethasone) therapy in relapsed or refractory multiple myeloma. Blood. 2013; 122:1940.

17. Jatin J. Shah, Edward A. Stadtmauer, Rafat Abonour, Adam D Cohen, William I. Bensinger, Cristina Gasparetto, Jonathan L. Kaufman, Suzanne Lentzsch, Dan T. Vogl, Robert Z. Orlowski, Erica L. Kim, Marti B. McKinley, Brian G M Durie. A Multi-Center Phase II Trial of Carfilzomib and Pomalidomide with Dexamethasone (CarPom-d) in Patients with Relapsed/Refractory Multiple Myeloma. Blood. 2012; 120.

18. Shah JJ, Stadtmauer EA, Abonour R, Cohen AD, Bensinger WI, Gasparetto C, Kaufman JL, Lentzsch S, Vogl DT, Gomes CL, Pascucci N, Smith DD, Orlowski RZ, et al. Carfilzomib, pomalidomide, and dexamethasone for relapsed or refractory myeloma. Blood. 2015;126:2284-90.

19. Jatin J. Shah, Edward A. Stadtmauer, Rafat Abonour, Adam D. Cohen, William Bensinger, Cristina Gasparetto, Jonathan L. Kaufman, Suzanne Lentzsch, Dan T. Vogl, Robert Z. Orlowski, Erica L. Kim, Natalia Bialas, David D. Smith, et al. Phase I/II dose expansion of a multicenter trial of carfilzomib and pomalidomide with dexamethasone (Ca-Pom-d) in patients with relapsed/refractory multiple myeloma. Blood. 2013; 122:690.

20. Larocca A, Montefusco V, Bringhen S, Rossi D, Crippa C, Mina R, Galli M, Marcatti M, La Verde G, Giuliani N, Magarotto V, Guglielmelli T, Rota-Scalabrini D, et al. Pomalidomide, cyclophosphamide, and prednisone for relapsed/refractory multiple myeloma: a multicenter phase 1/2 open-label study.Blood. 2013;122:2799-806.

21. MQ Lacy, B LaPlant, P Richardson, A Jakubowiak, K Laumann, S Kumar, MA Gertz, SR Hayman, F Buadi, A Dispenzieri, JA Lust, PKapoor, $\mathrm{N}$ Leung, et al. Pomalidomide, Bortezomib and Dexamethasone (PVD) for Patients with Relapsed Lenalidomide Refractory Multiple Myeloma (MM). Blood. 2014; 124.

22. Joseph R. Mikhael, Vivek Roy, Paul G. Richardson, Andrzej Jakubowiak, Kristina Laumann, Betsy R. LaPlant, Angela Dispenzieri, S. Vincent Rajkumar, Rafael Fonseca, Nelson Leung, Francis Buadi, P. Leif Bergsagel, Robert Kyle, et al. A phase I/II trial of pomalidomide, bortezomib and dexamethasone in patients with relapsed or refractory multiple myeloma. Blood. 2013; 122:1940.

23. Paul G. Richardson, Craig C. Hofmeister, David Siegel, Sagar Lonial, Jacob P. Laubach, Yvonne A. Efebera, David H. Vesole, Ajay K. Nooka, Jacalyn Rosenblatt, Noopur Raje, Mohamed H. Zaki, Ye Hua, Yan Li, Sheetal Shah, et al. MM-005: A Phase 1 Trial Of Pomalidomide, Bortezomib, and Low-Dose Dexamethasone (PVD) In Relapsed and/Or Refractory Multiple Myeloma (RRMM). Blood. 2013; 122.

24. Vij R, Siegel DS, Jagannath S, Jakubowiak AJ, Stewart AK, McDonagh K, Bahlis N, Belch A, Kunkel LA, Wear S, Wong AF, Wang M. An open-label, single-arm, phase 2 study of single-agent carfilzomib in patients with relapsed and/or refractory multiple myeloma who have been previously treated with bortezomib.Br J Haematol. 2012;158:739-48.

25. Vij R, Wang M, Kaufman JL, Lonial S, Jakubowiak AJ, Stewart AK, Kukreti V, Jagannath S, McDonagh KT, Alsina M, Bahlis NJ, Reu FJ, Gabrail NY, et al. An open-label, single-arm, phase 2 (PX-171-004) study of single-agent carfilzomib in bortezomib-naive patients with relapsed and/ or refractory multiple myeloma. Blood. 2012;119:5661-70.

26. Siegel DS, Martin T, Wang M, Vij R, Jakubowiak AJ, Lonial S, Trudel S, Kukreti V, Bahlis N, Alsina M, Chanan-Khan A, Buadi F, Reu FJ, et al. A phase 2 study of single-agent carfilzomib (PX-171-003-A1) in patients with relapsed and refractory multiple myeloma. Blood. 2012;120:2817-25.

27. Jagannath S, Vij R, Stewart AK, Trudel S, Jakubowiak AJ, Reiman T, Somlo G, Bahlis N, Lonial S, Kunkel LA, Wong A, Orlowski RZ, Siegel DS. An open-label single-arm pilot phase II study (PX-171-003-A0) of low-dose, single-agent carfilzomib in patients with relapsed and refractory multiple myeloma.Clin Lymphoma Myeloma Leuk. 2012;12:310-8. 
28. Jakubowiak AJ, Siegel DS, Martin T, Wang M, Vij R, Lonial S, Trudel S, Kukreti V, Bahlis N, Alsina M, ChananKhan A, Buadi F, Reu FJ, et al.Treatment outcomes in patients with relapsed and refractory multiple myeloma and high-risk cytogenetics receiving single-agent carfilzomib in the PX-171-003-A1 study. Leukemia. 2013;27:2351-6.

29. Badros AZ, Vij R, Martin T, Zonder JA, Kunkel L, Wang Z, Lee S, Wong AF, Niesvizky R. Carfilzomib in multiple myeloma patients with renal impairment: pharmacokinetics and safety.Leukemia. 2013;27:1707-14.

30. Papadopoulos KP, Siegel DS, Vesole DH, Lee P, Rosen ST, Zojwalla N, Holahan JR, Lee S, Wang Z, Badros A. Phase I study of 30-minute infusion of carfilzomib as single agent or in combination with low-dose dexamethasone in patients with relapsed and/or refractory multiple myeloma.J Clin Oncol. 2015;33:732-9.

31. Hájek R, Bryce R, Ro S, Klencke B, Ludwig H. A study of carfilzomib vs. best supportive care in subjects with relapsed and refractory multiple myeloma (FOCUS). ClinicalTrials.gov. Available at: http://clinicaltrials.gov/ show/NCT01302392.

32. Lendvai N, Hilden P, Devlin S, Landau H, Hassoun H, Lesokhin AM, Tsakos I, Redling K, Koehne G, Chung DJ, Schaffer WL, Giralt SA. A phase 2 single-center study of carfilzomib $56 \mathrm{mg} / \mathrm{m} 2$ with or without low-dose dexamethasone in relapsed multiple myeloma. Blood. 2014;124:899-906.

33. Berdeja JG, Hart LL, Mace JR, Arrowsmith ER, Essell JH, Owera RS, Hainsworth JD, Flinn IW.Phase I/II study of the combination of panobinostat and carfilzomib in patients with relapsed/refractory multiple myeloma. Haematologica. 2015;100:670-6.

34. Berenson JR, Hilger JD, Yellin O, Dichmann R, PatelDonnelly D, Boccia RV, Bessudo A, Stampleman L, Gravenor D, Eshaghian S, Nassir Y, Swift RA, Vescio RA. Replacement of bortezomib with carfilzomib for multiple myeloma patients progressing from bortezomib combination therapy. Leukemia. 2014;28:1529-36.

35. Niesvizky R, Martin TG, Bensinger WI, Alsina M, Siegel DS, Kunkel LA, Wong AF, Lee S, Orlowski RZ, Wang M. Phase Ib dose-escalation study (PX-171-006) of carfilzomib, lenalidomide, and low-dose dexamethasone in relapsed or progressive multiple myeloma. Clin Cancer Res. 2013;19:2248-56.

36. Stewart AK, Rajkumar SV, Dimopoulos MA, Masszi T, Špička I, Oriol A, Hájek R, Rosiñol L, Siegel DS, Mihaylov GG, Goranova-Marinova V, Rajnics P, Suvorov A, et al. Carfilzomib, lenalidomide, and dexamethasone for relapsed multiple myeloma. N Engl J Med. 2015;372:142-52.

37. Wang M, Martin T, Bensinger W, Alsina M, Siegel DS, Kavalerchik E, Huang M, Orlowski RZ, Niesvizky R. Phase 2 dose-expansion study (PX-171-006) of carfilzomib, lenalidomide, and low-dose dexamethasone in relapsed or progressive multiple myeloma. Blood. 2013;122:3122-8.
38. James R. Berenson, Leonard Klein, Robert M. Rifkin, Priti Patel, MD, Sandra Dixon, Ying Ou, Alan Cartmell. Results of the dose-escalation portion of a phase $1 / 2$ study (CHAMPION-1) investigating weekly carfilzomib in combination with dexamethasone for patients with relapsed or refractory multiple myeloma. J Clin Oncol. 2014;32:8594.

39. Meletios A. Dimopoulos, Philippe Moreau, Antonio Palumbo, Douglas E Joshua, Ludek Pour, Roman Hajek, Thierry Facon, Heinz Ludwig, Albert Oriol, Hartmut Goldschmidt, Laura Rosinol, Jan Straub, Aleksandr Suvorov, et al. Carfilzomib and dexamethasone $(\mathrm{Kd})$ vs bortezomib and dexamethasone (Vd) in patients (pts) with relapsed multiple myeloma (RMM): Results from the phase III study ENDEAVOR. J Clin Oncol. 2015.

40. Jonathan L. Kaufman, Todd Zimmerman, Cara A Rosenbaum, Ajay K. Nooka, Leonard T Heffner, R. Donald Harvey, Charise Gleason, Colleen Lewis, Cathy Sharp, Kenisha W Barron, Sagar Lonial. Phase I study of the combination of carfilzomib and panobinostat for patients with relapsed and refractory myeloma: a multicenter MMRC clinical trial [abstract]. Haematologica. 2013;98: Abstract 322.

41. Vesole DH, Bilotti E, Richter JR, McNeill A, McBride L, Raucci L, Anand P, Bednarz U, Ivanovski K, Smith J, Batra V, Aleman A, Sims T, et al. Phase I study of carfilzomib, lenalidomide, vorinostat, and dexamethasone in patients with relapsed and/or refractory multiple myeloma. Br J Haematol. 2015;171:52-9.

42. Bladé J, Samson D, Reece D, Apperley J, Björkstrand B, Gahrton G, Gertz M, Giralt S, Jagannath S, Vesole D. Criteria for evaluating disease response and progression in patients with multiple myeloma treated by high-dose therapy and haemopoietic stem cell transplantation. Br J Haematol. 1998;102: 1115-1123.

43. Richardson PG, Barlogie B, Berenson J, Singhal S, Jagannath S, Irwin D, Rajkumar SV, Srkalovic G, Alsina M, Alexanian R, Siegel D, Orlowski RZ, Kuter D, et al. A phase 2 study of bortezomib in relapsed, refractory myeloma. N Engl J Med. 2003;348: 2609-2617.

44. Durie BG, Harousseau JL, Miguel JS, Bladé J, Barlogie B, Anderson K, Gertz M, Dimopoulos M, Westin J, Sonneveld P, Ludwig H, Gahrton G, Beksac M, et al. International Myeloma Working Group. International uniform response criteria for multiple myeloma. Leukemia. 2006;20:14671473.

45. Berlin JA, Laird NM, Sacks HS, Chalmers TC. A comparison of statistical methods for combining event rates from clinical trials. Stat Med. 1989;8:141-51.

46. Rychack E, Mendy D, Miller K. Overcoming resistance; the use of pomalidomide (POM) and dexamethasone (DEX) in re-sensitizing lenalidomide (LEN)-resistant multiple myeloma (MM) cells. Haematologica. 2011;96:S126. Abstract P-328. 
47. Mitsiades N, Mitsiades CS, Poulaki V, Chauhan D, Richardson PG, Hideshima T, Munshi NC, Treon SP, Anderson KC. Apoptotic signaling induced by immunomodulatory thalidomide analogs in human multiple myeloma cells: therapeutic implications. Blood. 2002;99:4525-4530. 